\title{
Large Hemorrhagic Cerebral Venous Infarction due to Deep Brain Stimulation Leads Placement: Report of 2 Cases
}

\author{
Michał SOBSTYL ${ }^{1}$, Grażyna BRZUSZKIEWICZ-KUŹMICKA², Marta ALEKSANDROWICZ ${ }^{3}$, Tomasz PASTERSKI ${ }^{1}$ \\ ${ }^{1}$ Institute of Psychiatry and Neurology, Department of Neurosurgery, Warsaw, Poland \\ ${ }^{2}$ Academy of Physical Education in Warsaw, Department of Rehabilitation, Poland \\ ${ }^{3}$ Bródno Regional Hospital, Department of Neurosurgery, Warsaw, Poland \\ Corresponding author: Michał SOBSTYL mrsob@op.pl
}

\section{ABSTRACT}

The true incidence of hemorrhagic venous infarctions in deep brain stimulation (DBS) procedures is very difficult to determine. These hemorrhagic venous complications are very rare and often grouped as all hemorrhagic complications. We report the clinical cases of 2 patients with Parkinson's disease (PD) who received unilateral globus pallidus DBS and developed hemorrhagic venous infarctions. In these 2 patients a small injury to a dural outflow venous structure or a superficial brain vein resulted in hemorrhagic venous infarctions. We present the management of these rare complication with detailed radiologic follow-up. The first patient made a full recovery but the second patient deceased 5 months after DBS surgery due to aspiration pneumonia. We stress that careful planning of a stereotactic trajectory reduces significantly hemorrhagic complications in DBS surgery but not fully exclude some side effects like venous hemorrhagic infarctions which may result in prolong hospitalization or death.

KEYWORDS: Hemorrhagic venous infarction, Parkinson's disease, Pallidal stimulation, Deep brain stimulation, Globus pallidus

\section{INTRODUCTION}

L arge hemorrhagic cerebral venous infarction occurring after placement of leads for deep brain stimulation (DBS)

has rarely been reported $(4,5)$. The reported incidence of symptomatic hemorrhagic complications of DBS procedures is typically low $(1,3)$. Most reports on the incidence of hemorrhagic complications among patients undergoing DBS therapy did not differentiate patients on the basis of the etiology of intracerebral hemorrhage $(4,5)$. Relevant studies on clinical outcomes of venous infarction caused by DBS lead placement have included patients with varying manifestations ranging from only visible cerebral edema around the implanted DBS lead on postoperative computed tomography (CT) images to well-defined hemorrhagic cerebral venous infarction. In this case report, we present the outcomes of two patients with Parkinson's disease (PD) who developed a large hemorrhagic cerebral infarction after placement of DBS lead. Moreover, we discuss the management of this rare complication in these two patients and describe the detailed radiologic follow-up and long-term outcomes.

\section{CASE REPORTS}

\section{Case 1}

A 61-year-old woman with a history of PD since 13 years was admitted to the Neurosurgical Department for DBS therapy. Physical examination in off-medication state revealed bilateral bradykinesia and rigidity and severe resting tremor that was more pronounced on the right side. In the on-medication state, she exhibited severe levodopa-induced dyskinesia, predominantly on the right side. She was scheduled for staged bilateral DBS lead placement, first in the left globus pallidus internus (GPi) with the aim to ameliorate the severe disabling right-sided dyskinesia. On preoperative assessment, her total Unified Parkinson's Disease Rating Scale (UPDRS) score in off-medication and on-medication state was 103 and 34,

\begin{tabular}{llll}
\hline Michał SOBSTYL & (1) : 0000-0002-2684-3757 & Marta ALEKSANDROWICZ (1) : 0000-0002-7288-3653 \\
Grażyna BRZUSZKIEWICZ-KUŹMICKA (D) : 0000-0003-3590-6470 & Tomasz PASTERSKI & (1) : 0000-0003-0619-6422
\end{tabular}


respectively. The score for the motor part of UPDRS (part III) was 45 in the off-medication state and 14 in the on-medication state. Written informed consent of the patient was obtained prior to surgery.

In brief, stereotactic procedures were performed under local anesthesia using Leksell G stereotactic head frame. The initial stereotactic coordinates of the target - the posteroventrolateral $\mathrm{GPi}$-were 20 to 22 lateral, 2 to $4 \mathrm{~mm}$ anterior to, and 3 to 4 $\mathrm{mm}$ below the midcommissural point, as determined by T2weighted MRI images using stereotactic surgical planning software (Framelink, Shealth Station, Medtronic, Minneapolis, MN, USA). This planning software allows for adjustment to the individual patient's anatomy. The target was set just dorsal and lateral to the optic tract in the posteroventrolateral part of the GPi. A safe stereotactic trajectory through the middle frontal gyrus that avoided vascular surface structures, sulci, and the lateral ventricle was chosen to the left GPi. During dural opening, only mild venous bleeding was encountered, which was stopped using Surgicel. No intraoperative micorecording was obtained. A DBS electrode (St. Jude Medical) was implanted using a guiding cannula. The depth of the implanted DBS lead was checked by fluoroscopy. The macro stimulation through implanted DBS followed. Intraoperative stereotactic CT revealed appropriate lead placement with no signs of infarction or bleeding (Figure 1AF). Implantable pulse generator placement was postponed for the next morning. Unfortunately, $8 \mathrm{~h}$ after DBS lead placement, the patient experienced difficulty in finding the right words. Postoperative CT revealed a large subcortical hemorrhagic venous infarct with displacement of the DBS lead. The patient's consciousness level was intact. She developed partial dysarthria that completely resolved over the next 6 weeks. After 2 months, an implantable pulse generator (IPG) (Libra, St. Jude) was implanted in the left subclavicular region.

After turning on and programming the left IPG, her UPDRS motor scores in off-medication and on-medication state were 32 and 12, respectively. After switching off the left IPG, UPDRS motor scores were 41 and 14, respectively. Subsequently, right DBS was performed on her request. The preoperative T2-weighted MRI as well as the intraoperative CT showed appropriate location of the previously displaced DBS lead (Figure 1A-F). The right-sided DBS procedure was uneventful,

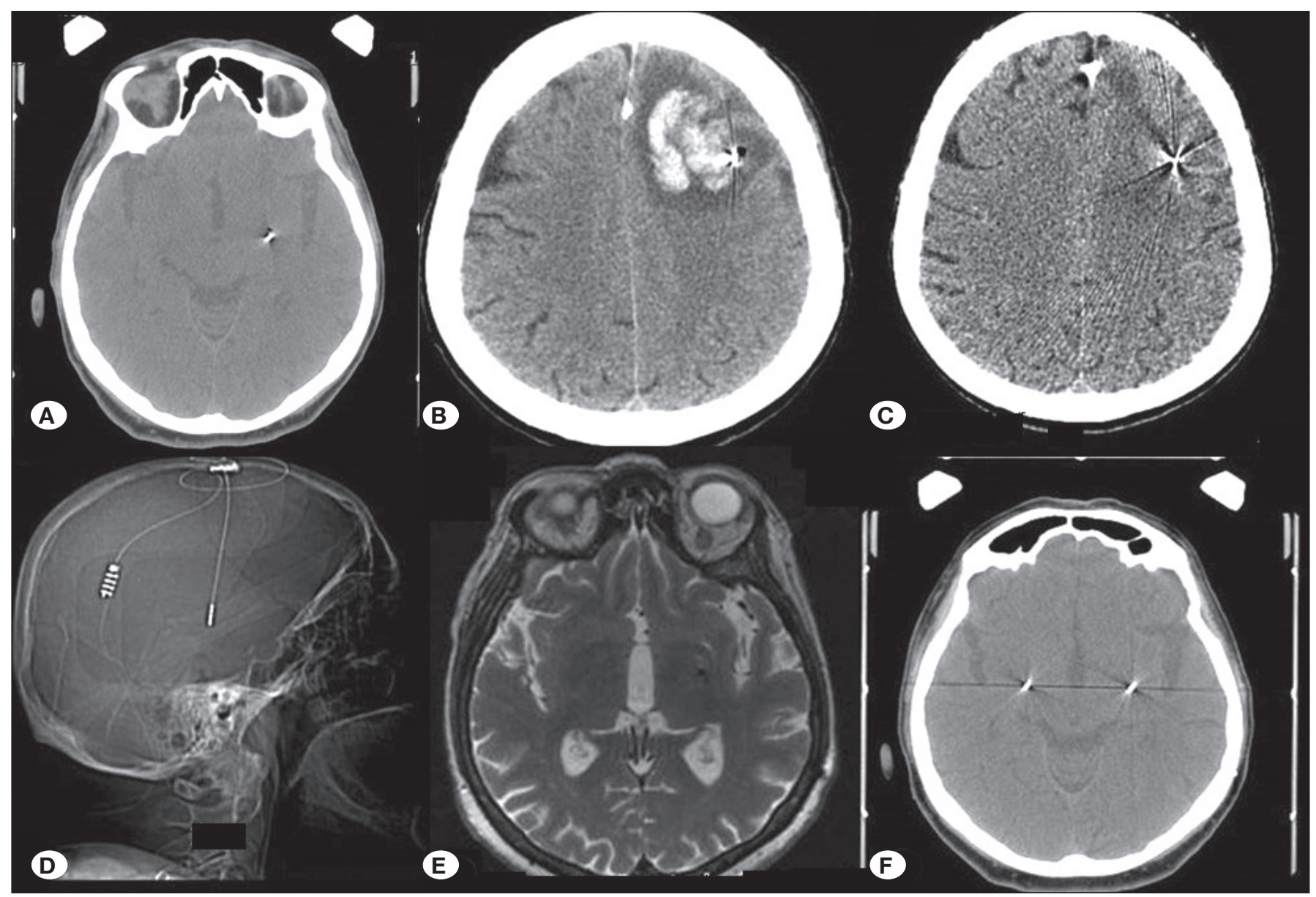

Figure 1: A) Stereotactic intraoperative computed tomography (CT) image showing the deep brain stimulation lead in the left globus pallidus pars interna, B) the repeated CT depicting a large hemorrhagic venous infarction surrounding the left DBS electrode in the left frontal region, C) the controlled CT showing complete resolution of venous infarction, D) the skull CT with implanted DBS electrode, E) an $\mathrm{MRI}$ taken before contralateral DBS surgery presenting DBS electrode in the left GPi, F) both electrodes implanted within GPi bilaterally. 
and the intraoperative CT showed both DBS leads in the GPi bilaterally (Figure 1A-F). She developed local inflammation around the surgical wound in the right subclavicular region. The IPG was relocated to the right abdominal region with no subsequent complications. Four years after the initial surgery and 2 years after bilateral GPi DBS, her UPDRS motor scores during bilateral GPi stimulation were reduced to 28 and 10 in the off-medication and on-medication state, respectively. After switching off both IPGs at the 4 year follow-up, her UPDRS motor scores in the off-medication and on-medication state were 48 and 17, respectively. Currently, she is able to independently perform her activities of daily living and does not experience severe and disabling bilateral dyskinesia when both IPGs are switched on.

\section{Case 2}

A 72-year-old man with a history of PD since 12 years was scheduled for right GPi DBS procedure. His PD symptoms were pronounced predominantly on the left side, and he exhibited tremor in the left upper extremity in the off-medication state. He also had incapacitating levodopa-induced dyskinesia only in the left extremities and painful early morning dystonia in the left foot. On preoperative assessment, his off-medication and onmedication total UPDRS scores were 50 and 32, respectively. His UPDRS motor scores were 32 and 16, respectively. He signed the written informed consent before DBS procedure. He underwent right-sided GPi DBS as described for the first patient. After pia coagulation and introduction of the guiding cannula to the target, we observed venous bleeding from the subdural space anterior to the burr hole. Introduction of Surgicel anteriorly completely stopped the venous bleeding. Intraoperative stereotactic CT showed the DBS lead in the appropriate place with no signs of intracranial bleeding (Figure 2A-F). An IPG (Activa 37603, Medtronic, Minneapolis, MN, USA) was implanted and connected to the DBS lead. Twentysix hours after the surgery, the patient became somnolent. CT revealed a large hemorrhagic venous infarct in the right frontal region with clearly visible displacement of the DBS lead (Figure 2A-F). Urgent frontal craniotomy was performed to evacuate the hematoma. Postoperative CT showed less midline shift and less displaced DBS lead as compared with that in the preoperative CT. The patient's status improved, and

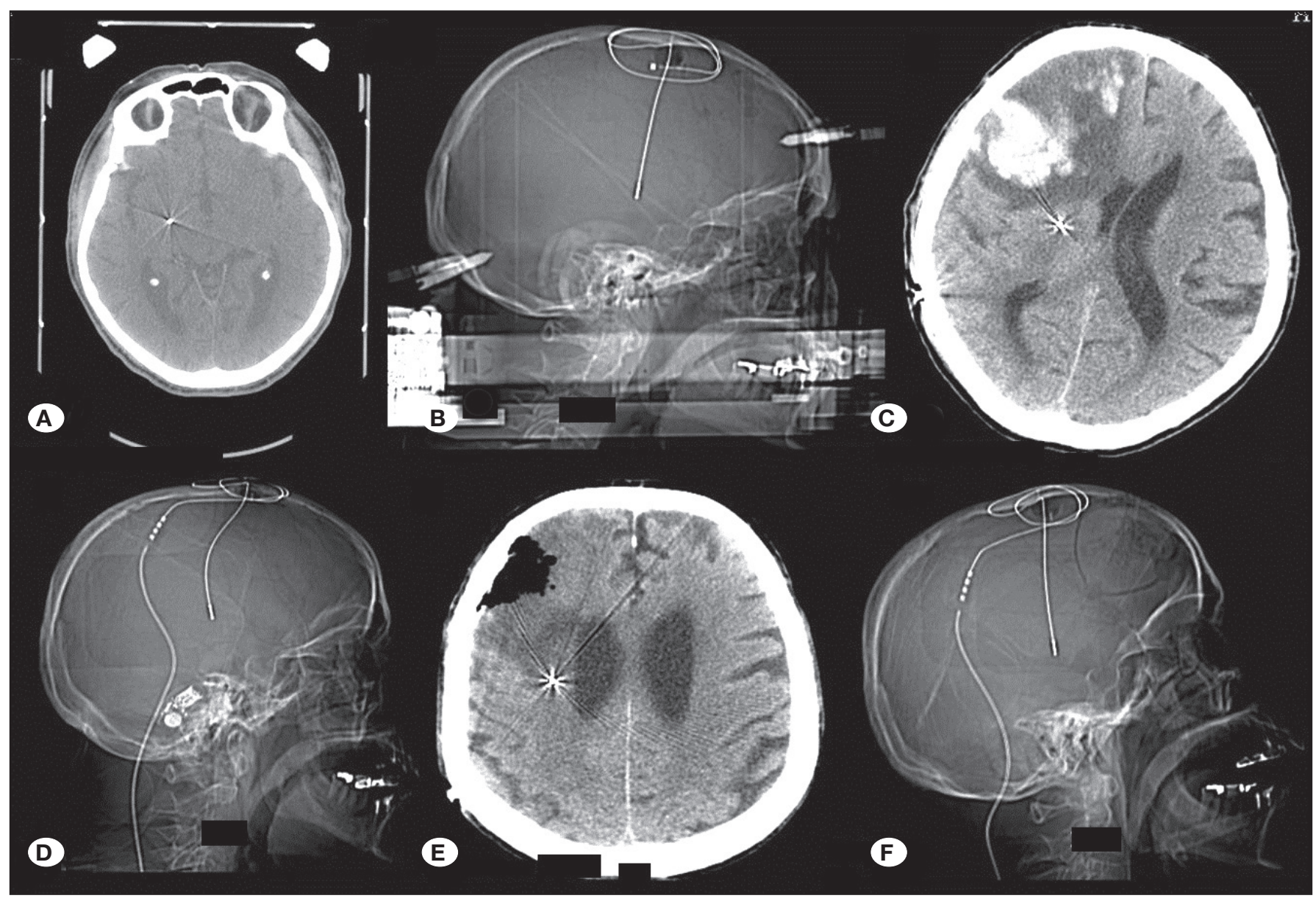

Figure 2: A) Stereotactic intraoperative computed tomography (CT) image showing the deep brain stimulation lead in the right globus pallidus pars interna, B) the lateral skull CT showing the DBS lead in place, C) the repeated CT depicting a large hemorrhagic venous infarction located anterior to the right implanted DBS electrode covering the whole right frontal region, D) the lateral skull CT presenting the displacement of DBS lead due to large hemorrhagic venous infarction in the right frontal lobe, E) the controlled CT after hematoma evacuation, F) the lateral skull CT showing the less displacement of DBS lead. 
he was able to perform his daily activities independently. $\mathrm{He}$ was transferred to a regional rehabilitation unit. He developed severe urinary tract infection and required assistance in activities of daily living. After 2 months, he was discharged home, where he received supportive care and some help with daily activities. There was considerable amelioration of left-sided dyskinesia. Unfortunately, the patient developed aspiration pneumonia and passed away 5 months after DBS surgery.

\section{DISCUSSION}

The true incidence of venous infarction and hemorrhagic venous infarction associated with DBS procedures is very difficult to determine. These hemorrhagic venous complications are very rare and are often grouped with other hemorrhagic complications in the reported series (5). Umumera et al. reported one patient with typical cerebral venous infarction and two patients with subcortical hemorrhage. One of these patients had a normal postoperative CT but developed mild permanent hemiparesis due to a large venous hemorrhagic infarction that was visible on repeat CT performed 6 hours later (5). We wish to highlight that venous hemorrhagic complications and venous infarction in the setting of DBS exhibit a unique postoperative course and demand further attention. Lack of routine postoperative CT assessment of patients after DBS surgery is a major reason for the ambiguity surrounding the true incidence of these complications. Small venous infarctions with no concomitant hemorrhage around the DBS lead may be missed (3).

During DBS procedure, we routinely perform intraoperative stereotactic CT after placement of the lead to ensure its proper localization and to exclude any intracranial hemorrhage before proceeding with general anesthesia and IPG placement. There after, if the postoperative course is uneventful, no another additional CT is performed. Theoretically, small venous infarct or small venous hemorrhage can escape recording (3). Similar to other authors, we would like to highlight that any injury to superficial venous structures can result in venous infarction or venous hemorrhagic infarction (1). We routinely perform merging of MR and contrast-enhanced CT images to avoid injury during preoperative trajectory planning. In the first patient, injury to intradural venous outflow resulted in a large left frontal venous infarction; in the second patient, the small pial opening for insertion of guiding cannula caused the deflection of the brain with subsequent tearing of a bridging superficial vein that led to a large venous hemorrhagic infarct in the right frontal region. According to Morishita et al., venous infarction/hemorrhage is a potentially avoidable complication of DBS surgery (1). In two out of their four cases of venous infarction, the planned trajectory traversed within $3 \mathrm{~mm}$ of a superficial vein. In the other two cases, no contrast-enhanced targeting images were obtained preoperatively. None of the patients presented by Morishita et al. required hematoma evacuation; the patient number 4 had a typical flame-shaped large hemorrhagic venous infarction in the left frontal region. Moreover, these authors have stressed that in addition to the superficial brain venous system, injury to venous channels located in the subdural or intradural space (the so-called venous lakes) in the path of stereotactic trajectory may also cause this complication (2).

In the event of a large hemorrhagic venous infarction that requires evacuation of the hematoma, the implanted DBS lead should not be removed. Interestingly, two DBS leads in our patients returned to their previous position after resolution of brain edema. Park et al. reported intracerebral hemorrhage in 13 out of 272 patients. In their series only one patient required hematoma evacuation with simultaneous removal of the DBS lead. Out of the 13 patients, 3 patients developed intracranial hemorrhage immediately after DBS, whereas 10 patients developed delayed $\mathrm{ICH}$ (3). This observation clearly highlights the notion that asymptomatic small venous infarction or even small venous hemorrhage may escape our attention (3). In our two patients, large hemorrhagic venous infarction led to prolonged hospitalization of the first patient and good neurological outcomes were achieved. However, the second patient experienced deterioration in general health and died.

\section{- CONCLUSION}

Large hemorrhagic venous infarction may lead to prolonged hospitalization or deterioration of neurological outcomes, including permanent neurological deficit or death. The risk of this complication can be minimized by careful stereotactic trajectory planning using neuronavigation software, but cannot be fully excluded. Our experience illustrates that even a small injury to dural outflow venous structure or a superficial brain vein injury may result in large hemorrhagic venous infarction. If hematoma evacuation is required, we advocate to leave the implanted DBS lead in situ, which can be used for permanent DBS after subsidence of brain edema. In our opinion, small asymptomatic venous infarction or venous hemorrhages may be missed.

\section{REFERENCES}

1. Morishita T, Okun MS, Burdick A, Jacobson CE, Foote KD: Cerebral venous infarction: A potentially avoidablecomplication of deep brain stimulation surgery. Neuromodulation 16:407413, 2013

2. Oka K, Rhoton AL, Barry M, Rodrigeuz R: Microsurgical anatomy of the superficial veins of the cerebrum. Neurosurgery 17:711-748, 1985

3. Park CK, Jung NY, Kim M, Chang JW: Analysis of delayed intracranial hemorrhage associated with deep brain stimulation surgery. World Neurosurgery 104:537-544, 2017

4. Sansur CA, Frysinger RC, Pouratian N, Fu KM, Bittl M, Oskouian RJ, Laws ER, Elias WJ: Incidence of symptomatic hemorrhage after stereotactic electrode placement. J Neurosurg 107:998-1003, 2007

5. Umumera A, Jaggi JL, Hurtig AD, Siderowf AD, Colcher A, Stern MB, Baltuch GH: Deep brain stimulation for movement disorders: Morbidity and mortality in 109 patients. J Neurosurg 98:779-784, 2003 